\title{
Evidence for a Carbon-Carbon Coupling Reaction to Proceed through a Planar-tetracoordinate Carbon Intermediate
}

\author{
Gerhard Erker*, Sabine Venne-Dunker, Gerald Kehr, Nina Kleigrewe, \\ Roland Fröhlich, Christian Mück-Lichtenfeld, Stefan Grimme*
}

\section{Supporting Material}

[Bis $\left(\eta^{5}\right.$-methylcyclopentadienyl)]-hex-2-en-4- $\left(\eta^{2}\right.$-in)-2-yl-3-[tris(pentafluorphenyl)borato]zirconium(IV) (3').

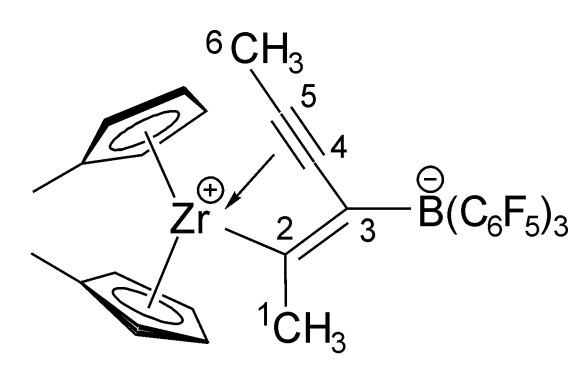

${ }^{1} \mathrm{H}-\mathrm{NMR}(200.1 \mathrm{MHz}, 300 \mathrm{~K}): \delta\left(\right.$ benzene-d $\left._{6}\right)=5.23$ (br m, 8H, Cp'), 2.26 (s, 3H, 1-H), 1.39 (br s, 6H, MeCp), 1.09 (s, $3 \mathrm{H}, 6-\mathrm{H}) ; \delta$ (toluene-d $\left.{ }_{8}\right)=5.36$ (br s, 2H, Cp'), 5.26 (br s, 4H, Cp'), 5.19 (br s, 2H, Cp'), 2.40 (s, 3H, 1-H), 1.44 (s, 6H, MeCp), 1.18 (s, 3H, 6-H). - ${ }^{19}$ F-NMR (benzene-d $6,282.4$ $\mathrm{MHz}, 300 \mathrm{~K}): \delta=-128.1$ (br, o-F $\left.\mathrm{F}_{1,3}\right),-139.9,-130.7\left(\mathrm{o}-\mathrm{F}_{2}\right)$, -159.9 (br, p-F $\left.\mathrm{F}_{1,3}\right),-160.2\left(\mathrm{p}-\mathrm{F}_{2}\right),-163.8\left(\mathrm{~m}-\mathrm{F}_{2}\right),-164.2$ (br, m$\left.\mathrm{F}_{1,3}\right),-166.3\left(\mathrm{~m}-\mathrm{F}_{2}\right)$. - ${ }^{11} \mathrm{~B}\left\{{ }^{1} \mathrm{H}\right\}-\mathrm{NMR}$ (benzene-d $\left.\mathrm{d}_{6}, 64.2 \mathrm{MHz}, 300 \mathrm{~K}\right): \delta=-13.3\left(\mathrm{v}_{1 / 2}=4 \mathrm{~Hz}\right)$. Elemental analysis: $\mathrm{C}_{36} \mathrm{H}_{20} \mathrm{BF}_{15} \mathrm{Zr}$ (839.57) calc. C 51.49, H 2.38; found C 51.73, H 2.60. - X-ray crystal structure analysis of $\mathbf{3}^{\prime}$ :

Data of $4 \mathrm{a}$ at $213 \mathrm{~K}$.

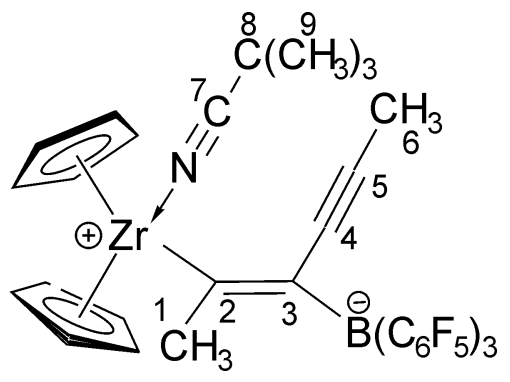

${ }^{1} \mathrm{H}-\mathrm{NMR}$ (toluene-d $8,599.9 \mathrm{MHz}, 213 \mathrm{~K}$ ): $\delta=5.54,5.27$ (each s, each 5H, Cp), 1.95 (s, 3H, 1-H), 1.21 (s, 3H, 6-H), 0.61 (s, 9H, 9H). $-{ }^{13} \mathrm{C}\left\{{ }^{1} \mathrm{H}\right\}$-NMR (toluene- $\mathrm{d}_{8}, 150.4 \mathrm{MHz}, 213 \mathrm{~K}$ ): $\delta=198.2$ (C2), 120.5 (C7), 110.8, 110.3 (Cp), 103.3 (C5), 68.2 (C4), 28.1 (C8), 25.2 (C1), 25.0 (C9), 7.5 (C6). The one dimensional ${ }^{13} \mathrm{C}$ data are extracted from the two dimensional GHSQC and 
GHMBC experiments. - GHSQC (toluene- $\mathrm{d}_{8}, 150.4 \mathrm{MHz} / 599.9 \mathrm{MHz}, 213 \mathrm{~K}$ ): $\delta^{13} \mathrm{C} / \delta^{1} \mathrm{H}=$ 110.8, 110.3/5.54, 5.27 (Cp/Cp), 25.2/1.95 (C1/1-H), 7.5/1.21 (C6/6-H), 25.0/0.61 (C9/9-H). GHMBC (toluene-d $\mathrm{d}_{8}, 150.4 \mathrm{MHz} / 599.9 \mathrm{MHz}, 213 \mathrm{~K}$ ): $\delta^{13} \mathrm{C} / \delta^{1} \mathrm{H}=198.2 / 1.95(\mathrm{C} 2 / 1-\mathrm{H})$, 120.5/0.61 (C7/9-H), 103.3/1.21 (C5/6-H), 78.2/1.21(C4/6-H), 28.1/0.61 (C8/9-H).

\section{Data of $4 \mathrm{~b}$ at $213 \mathrm{~K}$.}

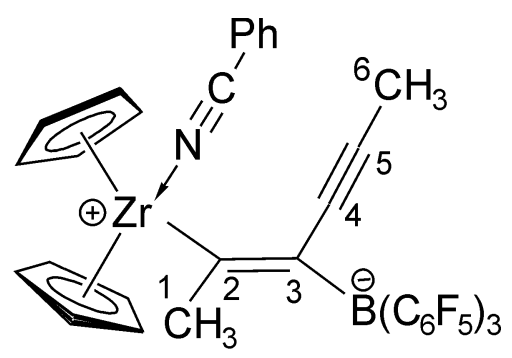

${ }^{1} \mathrm{H}-\mathrm{NMR}$ (toluene-d $8,599.9 \mathrm{MHz}, 213 \mathrm{~K}$ ): $\delta=6.67,6.49$ (each $\mathrm{m}, 5 \mathrm{H}, \mathrm{Ph}$ ), 5.62, 5.36 (each s, each 5H, $2 \mathrm{Cp}$ ), 1.96 (s, 3H, 1-H), 1.24 (s, 3H, 6-H). $-{ }^{13} \mathrm{C}\left\{{ }^{1} \mathrm{H}\right\}-\mathrm{NMR}$ (toluene-d $8,150.4 \mathrm{MHz}, 213$ $\mathrm{K}): \delta=198.0(\mathrm{C} 2), 131.8,131.8,128.4(\mathrm{Ph}), 111.6\left(\mathrm{Ph}_{\mathrm{ipso}}\right)$, 110.6, $110.3(\mathrm{Cp}), 106.4$ (C5), 63.8 (C4), 7.5 (C1), 1.4 (C6), (C3 resonance was not observed). - GHSQC (toluene- $\mathrm{d}_{8}, 150.4$ $\mathrm{MHz} / 599.9 \mathrm{MHz}, 213 \mathrm{~K}): \delta^{13} \mathrm{C} / \delta^{1} \mathrm{H}=131.8,131.8 .128 .4 / 6.67$, $6.49(\mathrm{Ph} / \mathrm{Ph}), 110.6,110.3 / 5.62,5.36$ (Cp/Cp), 7.5/1.96 (C1/1-H), 1.4/1.24 (C6/6-H). - GHMBC (toluene-d $\left.\mathrm{d}_{8}, 150.4 \mathrm{MHz} / 599.9 \mathrm{MHz}, 213 \mathrm{~K}\right): \delta^{13} \mathrm{C} / \delta^{1} \mathrm{H}=198.0 / 1.96(\mathrm{C} 2 / 1-\mathrm{H}), 131.8$, 128.4/6.67, $6.49(\mathrm{Ph} / \mathrm{Ph}), 111.6 / 6.49\left(\mathrm{Ph}_{\mathrm{ppso}} / \mathrm{Ph}\right), 110.6,110.3 / 5.36,5.62(\mathrm{Cp} / \mathrm{Cp}), 106.4 / 1.24$ (C5/6-H), 63.8/1.24 (C4/6-H).

\section{$\operatorname{Bis}\left(\eta^{5}\right.$-cyclopentadienyl)zirconacyclopentatrien (5).}

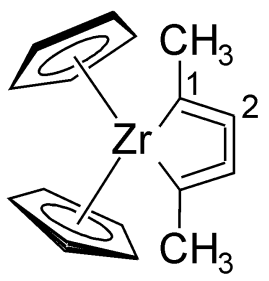

$+\left(\mathrm{CH}_{3}\right)_{3} \mathrm{CCN}-\mathrm{B}\left(\mathrm{C}_{6} \mathrm{~F}_{5}\right)_{3}$

${ }^{1} \mathrm{H}-\mathrm{NMR}$ (toluene-d $\mathrm{d}_{8}, 599.9 \mathrm{MHz}, 213 \mathrm{~K}$ ): $\delta=5.01$ (s, 10H, Cp), $2.77\left(\mathrm{~s}, 6 \mathrm{H}, \mathrm{CH}_{3}\right)$; borane-nitrile adduct $\delta=0.58\left(\mathrm{~s}, 9-\mathrm{H}, \mathrm{CH}_{3}\right)$. ${ }^{13} \mathrm{C}\left\{{ }^{1} \mathrm{H}\right\}$-NMR (toluene-d $\mathrm{d}_{8}, 150.4 \mathrm{MHz}$ ): $\delta=170.1(\mathrm{C} 1), 103.8(\mathrm{C} 2)$, $102.7(\mathrm{Cp}), 17.6\left(\mathrm{CH}_{3}\right)$; borane-nitrile adduct $\delta=119.1(\mathrm{CN}), 26.1$ $\left(\mathrm{CH}_{3}\right)$, the $\mathrm{CMe}_{3}$ carbon signal was not observed.

$\operatorname{Bis}\left(\eta^{5}\right.$-methylcyclopentadienyl)zirconacyclopentatrien (5').

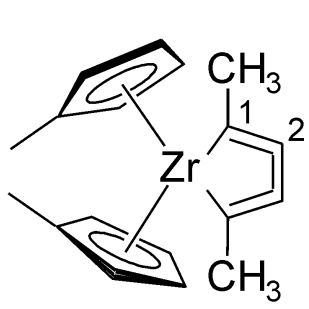

M.p.: $61{ }^{\circ} \mathrm{C} .{ }^{1}{ }^{1} \mathrm{H}-\mathrm{NMR}$ (benzene- $\mathrm{d}_{6}, 200.1 \mathrm{MHz}, 300 \mathrm{~K}$ ): $\delta=5.27,5.12$ (each m, each 4H, Cp'), $2.71\left(\mathrm{~s}, 6 \mathrm{H}, \mathrm{CH}_{3}\right) .-{ }^{13} \mathrm{C}\left\{{ }^{1} \mathrm{H}\right\}-\mathrm{NMR}$ (benzene- $\mathrm{d}_{6}$, $50.3 \mathrm{MHz}, 300 \mathrm{~K}): \delta=167.4(\mathrm{C} 1), 106.6$ (C2), 104.5, 99.0 (Cp'), 17.3 $\left(\mathrm{CH}_{3}\right), 12.1(\mathrm{MeCp})$. - IR (KBr): $\tilde{v}=2908(\mathrm{w}), 2855(\mathrm{vw}), 2365(\mathrm{w}), 2344$ 
(w), 1509 (w), 1494 (w), 1458 (m), 1374 (w), 1262 (m), 1088 (w), 1034 (m), 852 (m), 798 (s), $702(\mathrm{~m})$.

\section{Pivalonitrile Insertion Product 6a.}

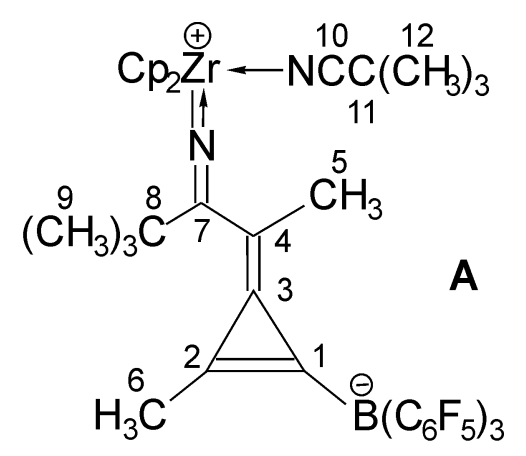

A yellow solid as a 4:1 mixture of two isomers (A major isomer; B minor isomer). Mp $238^{\circ} \mathrm{C}$ (decomp.). - Anal. calcd. for $\mathrm{C}_{44} \mathrm{H}_{34} \mathrm{BF}_{15} \mathrm{~N}_{2} \mathrm{Zr}$ (987.9) C 54.05, H 3.50, N 2.87; found $\mathrm{C}$ 54.00, H 3.81, N 3.35\%. - IR (KBr): $\tilde{v}=2982(\mathrm{~m}), 2941(\mathrm{w})$, $2877(\mathrm{w}), 2377(\mathrm{w}), 2212\left(\mathrm{vw}, \mathrm{v}_{\mathrm{C}} \mathrm{N}\right), 1811(\mathrm{w}), 1646(\mathrm{~m}), 1516$ (s), 1466 (vs), 1366 (m), 1279 (m), 1264 (m), 1092 (m), 1018 (m), 979 (s), 893 (w), 802 (s), $685(\mathrm{~m}) \mathrm{cm}^{-1}$. - ${ }^{1} \mathrm{H}-\mathrm{NMR}$ (benzene- $\mathrm{d}_{6}, 200.1,300 \mathrm{~K}$ ): A: $\delta=5.50(\mathrm{~s}, 10 \mathrm{H}, \mathrm{Cp}), 2.28$ (s,

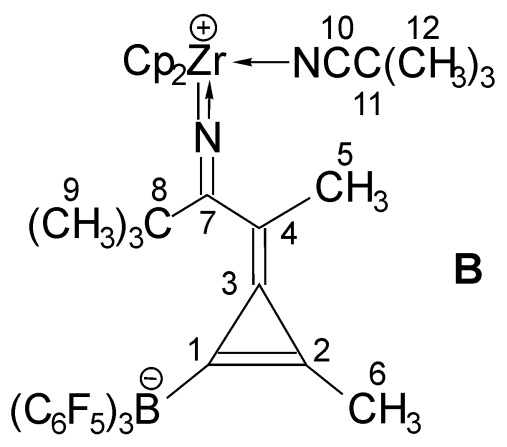
3H, 6-H), 1.74 (s, 3H, 5-H), 1.12 (s, 9H, 9-H), 0.77 (s, 9H, 12$\mathrm{H}) ; \mathbf{B}: \delta=5.58(\mathrm{~s}, 10 \mathrm{H}, \mathrm{Cp}), 2.31(\mathrm{~s}, 3 \mathrm{H}, 6-\mathrm{H}), 1.90(\mathrm{~s}, 3 \mathrm{H}, 5-$ $\mathrm{H}), 1.09$ (s, 9H, 9-H), 0.59 (s, 9H, 12-H). - ${ }^{1} \mathrm{H}-\mathrm{NMR}$ (toluene- $\mathrm{d}_{8}$, $599.9 \mathrm{MHz}, 213 \mathrm{~K}$ ): $\mathbf{A}: \delta=5.49$ (s, 10H, Cp), 2.23 (s, 3H, 6-H), 1.62 (s, 3H, 5-H), 1.07 (s, 9H, 9-H), $0.70(\mathrm{~s}, 9 \mathrm{H}, 12 \mathrm{H}) ; \mathbf{B}: \delta=$ 5.51 (s, 10H, Cp), 2.26 (s, 3H, 6-H), 1.77 (s, 3H, 5-H), 1.04 (s, 9H, 9-H), 0.65 (s, 9H, 12H). $-{ }^{13} \mathrm{C}\left\{{ }^{1} \mathrm{H}\right\}-\mathrm{NMR}$ (toluene- $\mathrm{d}_{8}, 150.4$ $\mathrm{MHz}, 213 \mathrm{~K}): \mathbf{A}: \delta=187.2(\mathrm{C} 7), 166.0(\mathrm{C} 1), 149.8(\mathrm{C} 3), 145.6$ $(\mathrm{C} 2), 148.9\left(\mathrm{dm},{ }^{1} \mathrm{~J}_{\mathrm{CH}}=244 \mathrm{~Hz}, \mathrm{o}-\mathrm{B}\left(\mathrm{C}_{6} \mathrm{~F}_{5}\right)_{3}\right), 139.5\left(\mathrm{dm},{ }^{1} \mathrm{~J}_{\mathrm{CH}}=246 \mathrm{~Hz}, \mathrm{p}-\mathrm{B}\left(\mathrm{C}_{6} \mathrm{~F}_{5}\right)_{3}\right), 147.7(\mathrm{dm}$, $\left.{ }^{1} \mathrm{~J}_{\mathrm{CH}}=258 \mathrm{~Hz}, \mathrm{~m}-\mathrm{B}\left(\mathrm{C}_{6} \mathrm{~F}_{5}\right)_{3}\right), 141.0(\mathrm{C} 10), 122.8\left(\mathrm{br}, \mathrm{i}-\mathrm{B}\left(\mathrm{C}_{6} \mathrm{~F}_{5}\right)_{3}\right), 110.3,110.2(\mathrm{Cp}), 83.8(\mathrm{C} 4)$, 38.9 (C8), 30.2 (C9), 28.1 (C11), 26.2 (C12), 22.5 (C6), 11.5 (C5); B: $\delta=187.2$ (C7), 166.0 (C1), 147.9 (C3), 145.4 (C2), 140.3 (C10), 111.6, 111.5 (Cp), 82.1 (C4), 41.5 (C8), 29.9 (C9), 29.4 (C11), 26.3 (C12), 21.3 (C6), 11.5 (C5). The ${ }^{13} \mathrm{C}$ resonances of $\mathrm{C} 1$ were not observed; the ${ }^{13} \mathrm{C}$ resonances of the $\mathrm{C}_{6} \mathrm{~F}_{5}$ unit were superposed. $-{ }^{11} \mathrm{~B}\left\{{ }^{1} \mathrm{H}\right\}$-NMR (toluene- $\mathrm{d}_{8}, 64.2 \mathrm{MHz}, 300$ $\mathrm{K}): \delta=-17.3\left(v_{1 / 2}=3 \mathrm{~Hz}\right) .{ }^{19} \mathrm{~F}-\mathrm{NMR}$ (toluene-d8, $\left.282.4 \mathrm{MHz}, 213 \mathrm{~K}\right): \mathbf{A}: \delta=-134.3(\mathrm{o}-$ $\left.\mathrm{B}\left(\mathrm{C}_{6} \mathrm{~F}_{5}\right)_{3}\right),-163.0\left(\mathrm{p}-\mathrm{B}\left(\mathrm{C}_{6} \mathrm{~F}_{5}\right)_{3}\right),-167.5\left(\mathrm{~m}-\mathrm{B}\left(\mathrm{C}_{6} \mathrm{~F}_{5}\right)_{3}\right) ; \mathbf{B}: \delta=-133.8\left(\mathrm{o}-\mathrm{B}\left(\mathrm{C}_{6} \mathrm{~F}_{5}\right)_{3}\right),-162.3(\mathrm{p}-$ $\left.\mathrm{B}\left(\mathrm{C}_{6} \mathrm{~F}_{5}\right)_{3}\right),-167.1\left(\mathrm{~m}-\mathrm{B}\left(\mathrm{C}_{6} \mathrm{~F}_{5}\right)_{3}\right)$. - GHSQC (toluene-d $\left.\mathrm{d}_{8}, 150.4 \mathrm{MHz} / 599.9 \mathrm{MHz}, 213 \mathrm{~K}\right): \mathbf{A}: \delta^{13} \mathrm{C} /$ $\delta^{1} \mathrm{H}=110.3,110.2 / 5.49(\mathrm{Cp} / \mathrm{Cp}), 30.2 / 1.07(\mathrm{C} 9 / 9-\mathrm{H}), 26.2 / 0.70(\mathrm{C} 12 / 12-\mathrm{H}), 22.5 / 2.23(\mathrm{C} 6 / 6-\mathrm{H})$, 11.5/1.62 (C5/5-H); B: $\delta^{13} \mathrm{C} / \delta^{1} \mathrm{H}=111.6,111.5 / 5.51(\mathrm{Cp} / \mathrm{Cp}), 29.9 / 1.04(\mathrm{C} 9 / 9-\mathrm{H}), 26.3 / 0.65$ (C12/12-H), 21.3/2.26 (C6/6-H), 11.5/1.77 (C5/5-H). - GHMBC (toluene-d $\mathrm{d}_{8}, 150.4 \mathrm{MHz} / 599.9$ MHz, $213 \mathrm{~K}): \mathbf{A}: \delta^{13} \mathrm{C} / \delta^{1} \mathrm{H}=187.2 / 1.62(\mathrm{C} 7 / 5-\mathrm{H}), 187.2 / 1.07(\mathrm{C} 7 / 9-\mathrm{H}), 166.0 / 2.23(\mathrm{C} 1 / 6-\mathrm{H})$, 149.8/2.23 (C3/6-H), 149.8/1.62 (C3/5-H), 145.6/2.23 (C2/6-H), 141.0/0.70 (C10/12-H), 
83.8/1.62 (C4/5-H), 38.9/1.07 (C8/9-H), 28.1/0.70 (C11/12-H); B: $\delta^{13} \mathrm{C} / \delta^{1} \mathrm{H}=187.2 / 1.77(\mathrm{C} 7 / 5-$ $\mathrm{H}), 187.2 / 1.04(\mathrm{C} 7 / 9-\mathrm{H}), 166.0 / 2.26(\mathrm{C} 1 / 6-\mathrm{H}), 147.9 / 2.26(\mathrm{C} 3 / 6-\mathrm{H}), 147.9 / 1.77$ (C3/5-H), 145.4/2.23 (C2/6-H), 140.3/0.65 (C10/12-H), 82.1/1.77 (C4/5-H), 41.5/1.04 (C8/9-H), 29.4/0.65 (C11/12-H).

\section{Benzonitrile Insertion Product 6b.}

Yield: 91\%. - IR (KBr): $\tilde{v}=3083$ (w), 2959 (w), 2907 (w), $2254\left(\mathrm{~m}, v_{\mathrm{C} \equiv \mathrm{N}}\right), 1822(\mathrm{~s}), 1642(\mathrm{~m})$,

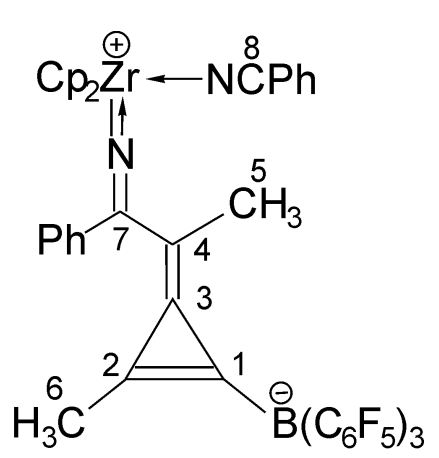

1579 (m), 1513 (vs), 1460 (vs), 1363 (s), 1276 (s), 1162 (m), 1091 (s), 1018 (w), 975 (vs), 806 (s), 685 (m) $\mathrm{cm}^{-1}$. - ${ }^{1} \mathrm{H}-\mathrm{NMR}$ (chloroform- $\left.\mathrm{d}_{1}, 200.1 \mathrm{MHz}, 300 \mathrm{~K}\right): \delta 7.65-7.50(\mathrm{~m}, 5 \mathrm{H}, \mathrm{Ph}), 7.35$ 7.15 (m, 5H, Ph), 6.10 (s, 10H, Cp), 1.50 (s, 3H, 5-H), 1.17 (s, 3H, 6H). - ${ }^{1} \mathrm{H}-\mathrm{NMR}$ (toluene-d $8,599.9 \mathrm{MHz}, 298 \mathrm{~K}$ ): $\delta$ 6.88, 6.79, 6.64 (each m, 10H, Ph), 5.52 (s, 10H, Cp), 1.85 (s, 3H, 5-H), 1.28 (s, 3H, 6-H). - ${ }^{1} \mathrm{H}-\mathrm{NMR}$ (toluene- $\mathrm{d}_{8}, 599.9 \mathrm{MHz}, 213 \mathrm{~K}$ ): $\delta$ 6.71, 6.63, 6.46 (each m, 10H, Ph), 5.39 (s, 10H, Cp), 2.09 (s, 3H, 5-H), 1.41 (s, 3H, 6-H). $-{ }^{13} \mathrm{C}\left\{{ }^{1} \mathrm{H}\right\}$-NMR (toluene- $\mathrm{d}_{8}, 150.4 \mathrm{MHz}, 213 \mathrm{~K}$ ): $\delta 181.6(\mathrm{C} 7)$, $173.5(\mathrm{C} 3), 153.7(\mathrm{C} 2), 149.0\left(\mathrm{dm},{ }^{1} \mathrm{~J}_{\mathrm{CH}}=241 \mathrm{~Hz}, \mathrm{o}-\mathrm{B}\left(\mathrm{C}_{6} \mathrm{~F}_{5}\right)_{3}\right), 145.7(\mathrm{C} 8), 139.6\left(\mathrm{dm},{ }^{1} \mathrm{~J}_{\mathrm{CH}}=\right.$ $\left.246 \mathrm{~Hz}, \mathrm{p}-\mathrm{B}\left(\mathrm{C}_{6} \mathrm{~F}_{5}\right)_{3}\right), 137.4\left(\mathrm{dm},{ }^{1} \mathrm{~J}_{\mathrm{CH}}=247 \mathrm{~Hz}, \mathrm{~m}-\mathrm{B}\left(\mathrm{C}_{6} \mathrm{~F}_{5}\right)_{3}\right), 133.9,133.5,132.8,130.6,129.8$, 129.5, 128.5, $127.8(\mathrm{Ph}), 112.6(\mathrm{Cp}), 87.6(\mathrm{C} 4), 15.9(\mathrm{C} 5), 9.0(\mathrm{C} 6)$. The $13 \mathrm{C}$ resonanzes of $\mathrm{C} 1$ and ipso-B $\left(\mathrm{C}_{6} \mathrm{~F}_{5}\right)_{3}$ were not observed. 


\section{X-ray crystal structure analysis:}

X-ray crystal structure analysis of $\mathbf{3}^{\prime}$ : formula $\mathrm{C}_{36} \mathrm{H}_{20} \mathrm{BF}_{15} \mathrm{Zr}, M=839.55$, colourless crystal $0.35 \times 0.15 \times 0.10 \mathrm{~mm}, a=15.6168(1), b=20.0336(2), c=20.2833(2) \AA, \beta=90.012(1)^{\circ}, V=$

6345.85(10) $\AA^{3}, \rho_{\text {calc }}=1.758 \mathrm{~g} \mathrm{~cm}^{-3}, \mu=4.63 \mathrm{~cm}^{-1}$, empirical absorption correction $(0.855 \leq T$

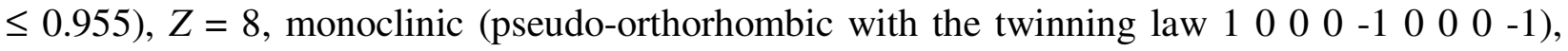
space group $P 2{ }_{1} / \mathrm{c}$ (No. 14$), \lambda=0.71073 \AA, T=198 \mathrm{~K}, \omega$ and $\varphi$ scans, 48557 reflections collected $( \pm h, \pm k, \pm l),[(\sin \theta) / \lambda]=0.65 \AA^{-1}, 13397$ independent $\left(R_{\text {int }}=0.059\right)$ and 11026 observed reflections $[I \geq 2 \sigma(I)], 964$ refined parameters, $R=0.038, w R^{2}=0.073$, max. residual electron density $0.37(-0.55)$ e $\AA^{-3}$, hydrogens calculated and refined as riding atoms, ratio of the twins refined to $0.629(1): 0.371(1)$, two almost identical molecules in the asymmetric unit.

Data set was collected with a Nonius KappaCCD diffractometer, equipped with a rotating anode generator Nonius FR591. Programs used: data collection COLLECT (Nonius B.V., 1998), data reduction Denzo-SMN (Z. Otwinowski, W. Minor, Methods in Enzymology 276, 307 (1997)), absorption correction SORTAV (R.H. Blessing, Acta Cryst. A 51, 33 (1995). R.H. Blessing, J. Appl. Cryst. 30, 421 (1997)), structure solution SHELXS-97 (G.M. Sheldrick, Acta Cryst. A 46, 467 (1990)), structure refinement SHELXL-97 (G.M. Sheldrick, Universität Göttingen, 1997), graphics SCHAKAL (E. Keller, Universität Freiburg, 1997).

Crystallographic data (excluding structure factors) for the structure reported in this paper have been deposited with the Cambridge Crystallographic Data Centre as supplementary publication CCCD-229224. Copies of the data can be obtained free of charge on application to The Director, CCDC, 12 Union Road, CambridgeCB2 1EZ, UK [fax: int. code +44(1223)336-033, e-mail: deposit@ccdc.cam.ac.uk].

Selected bond lengths and angles for the two independent molecules ( $\mathbf{3}^{\prime}$ ):

Zr-C2 2.172(3)/2.177(3), $\quad \mathrm{Zr}-\mathrm{C} 3 \quad 2.637(3) / 2.648(3), \quad \mathrm{Zr}-\mathrm{C} 4 \quad 2.374(3) / 2.378(3), \quad \mathrm{Zr}-\mathrm{C} 5$ 2.756(3)/2.680(3), Zr-C $\mathrm{Cp}_{\mathrm{p}}$ 2.461(4)-2.528(3)/2.459(3)-2.565(4), C1-C2 1.499(4)/1.495(4), C2-

C3 1.357(4)/1.359(4), B1-C3 1.684(5)/1.683(5), C3-C4 1.451(4)/1.461(5), C4C51.215(5)/1.200(5), C5-C6 1.465(5)/1.471(5); C2-Zr-C3 30.9(1)/30.8(1), C2-Zr-C4 64.0(1)/64.0(1), C2-Zr-C5 90.1(1)/90.6(1), C3-Zr-C4 33.2(1)/33.2(1), C3-Zr-C5 59.2(1)/59.8(1), C4-Zr-C5 26.1(1)/26.6(1), C1-C2-Zr 141.3(2)/140.6(2), C3-C2-Zr 93.8(2)/94.1(2), C1-C2-C3 124.9(3)/125.3(3), C2-C3-C4 118.7(3)/118.3(3), C2-C3-B 125.7(3)/124.7(3), C4-C3-B 115.4(3)/116.6(3), C3-C4-C5 176.6(3)/174.2(4), C3-C4-Zr 83.4(2)/83.6(2), C5-C4-Zr 94.8(2)/90.9(2), C4-C5-C6 172.6(4)/175.6(4). 


\section{DFT Calculations}

All quantum chemical calculations have been performed with the TURBOMOLE suite of programs $^{[a]}$. The structures have been fully optimised at the density functional (DFT) level employing the B-LYP functional ${ }^{[b]}$. A Gaussian AO basis of valence-triple-zeta quality including polarisation functions (TZVP) ${ }^{[c]}$ was used for heavy atoms, except for the $\mathrm{C}_{6} \mathrm{~F}_{5}$ substituents, for which a split-valence basis set with polarisation functions (SVP) ${ }^{[\mathrm{d}]}$ was used. One additional fpolarisation function $(\alpha=0.988993)$ from the TURBOMOLE basis set library and a relativistic pseudopotential (small core) ${ }^{[\mathrm{e}]}$ were employed for zirconium. The RI-approximation was used for the two-electron integrals ${ }^{[\mathrm{f}]}$.

[a] TURBOMOLE (Vers. 5.3): R. Ahlrichs, M. Bär, H.-P. Baron, R. Bauernschmitt, S. Böcker, M. Ehrig, K. Eichkorn, S. Elliott, F. Furche, F. Haase, M. Häser, H. Horn, C. Huber, U. Huniar, M. Kattannek, C. Kölmel, M. Kollwitz, K. May, C. Ochsenfeld, H. Öhm, A. Schäfer, U. Schneider, O. Treutler, M. von Arnim, F. Weigend, P. Weis, H. Weiss. Universität Karlsruhe 2000. ${ }^{[b]}$ A. D. Becke, Phys. Rev. A. 38, 3098 (1988). C. Lee, W. Yang, R. G. Parr, Phys. Rev. B 37, 785 (1988). ${ }^{[c]}$ A. Schäfer, C. Huber, R. Ahlrichs, J. Chem. Phys. 100, 5829 (1994). ${ }^{[d]}$ A. Schäfer, H. Horn, R. Ahlrichs, J. Chem. Phys. 97, 2571 (1992). ${ }^{[e]}$ D. Andrae, U. Haeussermann, M. Dolg, H. Stoll, H. Preuss, Theor. Chim. Acta 77, 123 (1990). ${ }^{[\mathrm{ff}]}$ K. Eichkorn, O. Treutler, H. Öhm, M. Häser, R. Ahlrichs, Chem. Phys. Letters 240, 283 (1995).

Tables: Cartesian coordinates of optimized structures (B-LYP/TZVP) in atomic units (a.u.)

3. (Energy: -2872.5526248 a.u.)

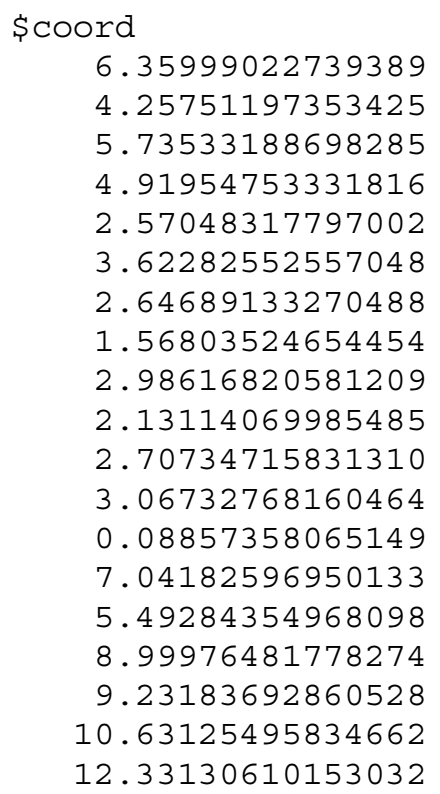

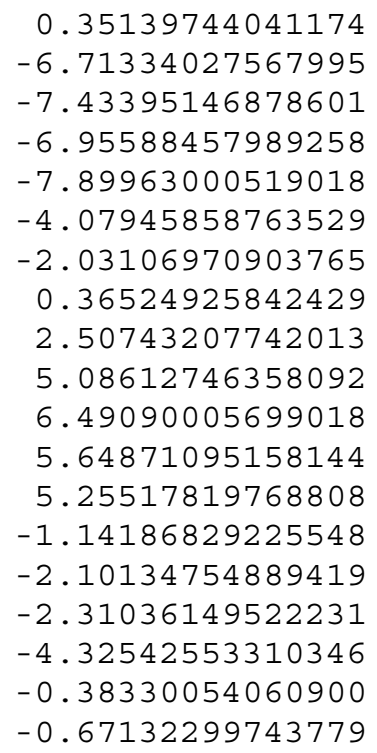

$\begin{array}{rl}-1.09894789756817 & \mathrm{zr} \\ -1.99786290177250 & \mathrm{C} \\ -0.72828905720218 & \mathrm{~h} \\ -3.95219023875082 & \mathrm{~h} \\ -1.74531494891128 & \mathrm{~h} \\ -1.44677381079465 & \mathrm{C} \\ -0.89225576309209 & \mathrm{C} \\ -0.25109515810617 & \mathrm{C} \\ 0.02159728171038 & \mathrm{C} \\ 0.78484607229860 & \mathrm{C} \\ -0.63485700918406 & \mathrm{~h} \\ 2.55738966560009 & \mathrm{~h} \\ 1.06894106293698 & \mathrm{~h} \\ 3.53784484248486 & \mathrm{C} \\ 4.47691396440712 & \mathrm{~h} \\ 2.09603961443778 & \mathrm{C} \\ 1.78779508296783 & \mathrm{~h} \\ 1.18126974404192 & \mathrm{C} \\ 0.07553933423489 & \mathrm{~h}\end{array}$


9.67400299027382

10.52848174129839

7. 47584629625769

6.35306231920199

9.50318372083306

11.48104092294667

7.59714171349426

7.86439668190128

5.28337959257561

3. 46467051931689

5. 76514856472400

4.38023523855265

8.36460303908414

9.33199243271769

$-1.72423274055682$

$-2.80949786021290$

$-2.49079410876641$

$-1.17973013196797$

$-3.41346784911053$

$-2.96449331580482$

$-4.85670102685974$

$-5.78965279776193$

$-5.28649793466340$

$-6.68345864220435$

$-4.28917315344828$

$-4.84694020872745$

$-1.68040961432977$

$-0.45164823211608$

0.60465103571791

$-0.16023503416177$

1.10029391542899

$-1.13712332914296$

$-0.88421441101595$

$-2.36960608829144$

$-3.29493870215045$

$-2.57599611107740$

$-3.69847738839745$

$-3.41223646362737$

$-2.66185508716985$

$-0.21885114613520$

$-4.30404956596046$

$-3.43205876257790$

$-6.87590829677647$

$-8.47749730922608$

$-7.74402178120540$

$-10.20352975741533$

$-6.02519398221653$

$-6.99913730620242$ \$end
1. 99052473769160

3. 82559513237007

1. 51136941245831

2. 91755685238039

1. 05137057110268

0.57949966315870

$-0.57926994584504$

$-2.52772623429617$

0.80281221598760

0.08372914436686

3. 29804402892549

4. 80033167496455

3. 44688030256666

5. 11395293422241

0.13938821808139

$-2.65057139266601$

$-3.42199691800510$

$-1.92069143804006$

$-5.70056084483762$

$-6.33398465255578$

$-7.30525398928333$

$-9.48532612996419$

$-6.60824599833268$

$-8.11515343056550$

$-4.31598969425055$

$-3.79184733514097$

0.58527717321293

$-1.17190365464091$

$-3.30300530584185$

$-0.89659246776103$

$-2.65271366949489$

1. 27099708077678

1. 58972038429007

3. 09800289737195

5. 20240479495973

2. 74193903907384

4.68154563261103

2. 18791328330622

3. 59457977118866

3. 56995627139521

5.13942656803794

6.45516890668750

5. 25642039567570

6. 69871259244487

3. 86183507892957

3. 94229663126022

2. 35678052868393

1. 01472833714600
2.03959719701606

1. 71050709447626

3. 52419698253055

4. 49974236739715

$-4.77453120499049$

$-4.52511320289564$

$-5.73820640574001$

$-6.32244674756574$

$-5.83549249519358$

$-6.44689758529170$

$-4.95146118009860$

$-4.84583351225737$

$-4.26395157088067$

$-3.56269369793366$

0.37484275805446

$-0.55657306344079$

$-3.09180276704609$

$-4.73555952196074$

$-4.10012081332026$

$-6.52947236196263$

$-2.54666294905076$

$-3.46140967720026$

$-0.01917715868750$

1. 48233623908469

0.91043428864819

3. 35300467306341

3. 46959056123727

5.06108125080243

4.05004798154524

7.68269664357342

9.03822770480737

8.87583651554732

11.38383882877604

7. 39445770464208

8.48700215422783

4.75887656587737

3. 49613248866110

$-1.29862707256073$

$-3.43094031616266$

$-4.27235091445211$

$-4.85245141196750$

$-6.85533882564874$

$-4.20914874704100$

$-5.55649287231015$

$-2.11739699863670$

$-1.46907314003796$

$-0.76209314073402$

1. 19814658413539
C

$\mathrm{h}$

C

$\mathrm{h}$

C

$\mathrm{h}$

C

$\mathrm{h}$

C

h

c

h

C

$\mathrm{h}$

b

C

C

f

C

f

C

f

C

f

C

f

C

C

f

C

f

C

f

C

f

C

f

C

C

f

C

f

C

f

C

f

C

f

\section{4. (Energy: -3005.3087637 a.u.)}

\$coord
6.35484389670216
2. 32572347693681
3. 67737174762549
2. 87171227310731

$-0.41813602540066$

$-7.40040831444258$

$-8.46602369948093$

$-7.61629196139735$
$-0.75861410935300$

$-2.34427213123418$

$-1.17692818038479$

$-4.33971823605404$ zr

$\mathrm{C}$

$\mathrm{h}$

h 
0.47033389296436

2. 14992390244351

2. 01463633899976

1. 10189043843581

2.87387019430735

2. 29381711209343

3. 00332221470800

3. 24475100884575

0.27813820811368

6. 21803433923285

4. 37598937505146

7. 92056100406454

7. 64606685210142

10.10459239133102

11.77452891261098

9.72755772636692

11.07224813492066

7. 31150991533842

6.48529094902286

9. 28824243338470

11.11163709741357

6.95539612080757

6.69295086287267

5.04545613735986

3. 06067010635016

6.17437528728270

5. 20171591974435

8.81040092299976

10.21782209582556

$-2.10661211570990$

$-3.58143751025545$

$-3.23962840537160$

$-1.66543575176869$

$-4.41609542171532$

$-3.92838316812290$

$-6.15040074544604$

$-7.33217778204688$

$-6.61174517908795$

$-8.28201931982638$

$-5.36407212019913$

$-5.99699026747905$

$-2.29897350433395$

$-1.49348260602973$

$-0.66355618595232$

$-1.44731333545551$

$-0.64409428446142$

$-2.20853523573895$

$-2.17072754327748$

$-2.98901735247111$

$-3.68103239537701$

$-2.99395449024800$

$-3.68348266241031$

$-3.37171583208942$

$-2.25010165310629$

0.20832306393920

$-3.50924839841842$

$-2.29039096469915$

$-6.05957096047144$
$-8.31061903382756$

$-4.76190563350528$

$-2.53904619309995$

$-0.09053986149534$

1. 69748283768273

4. 29619032474497

5. 75433235792322

4.61459561389176

4.65815536096090

$-3.45167960163923$

$-4.17069404141435$

$-4.57733531778725$

$-6.35751817559400$

$-3.01493167896429$

$-3.38563477944089$

$-0.89983356988129$

0.61109335221364

$-1.16644238547735$

0.11576900766650

$-1.14776218220351$

$-2.05366965342038$

$-2.38293584792046$

$-4.40177478747616$

$-0.49166323456090$

$-0.82609095426887$

1. 89793317183694

3. 69920513624752

1. 48941991898308

2. 94137635465804

0.14931660760089

$-2.33232591141788$

$-2.90183829459533$

$-1.45107610378256$

$-4.91835308413580$

$-5.37832904312633$

$-6.43965446459752$

$-8.36391652532000$

$-5.93186475762493$

$-7.36188207116283$

$-3.89771309812035$

$-3.52808009636860$

0.31907789610691

$-1.73986333024615$

$-3.91180630446252$

$-1.76216437962599$

$-3.82082188434815$

0.41253955326311

0.45568253581583

2. 54086185402074

4.66229085765542

2. 45678586551821

4. 66421801092718

2. 58316401963147

4.03001723861546

3. 66968220422719

5.95729448210378

7. 27961975496131

6.46761988161252
$-2.11226915634662$

$-1.58673956747571$

$-0.92239007104871$

$-0.08991961449519$

0.46755657575921

1. 45585943220392

0.15207230618442

3. 28284361506125

1. 73571217923317

3. 15837063847308

3. 69493999736360

1. 39185411779332

0.41445463349422

1. 22402543804349

0.09669618471827

2. 82987019666545

3. 15227969072863

4. 02557059531827

5.39333410507747

$-4.74826266211540$

$-4.51284060813389$

$-5.26060237262374$

$-5.50117043697336$

$-5.52209172101877$

$-5.90140866404338$

$-5.09845200116732$

$-5.16738621986572$

$-4.61358590121774$

$-4.28733514845429$

0.31505262904936

$-0.93515188769168$

$-3.52172006844588$

$-4.96807855596732$

$-4.79275887178531$

$-7.25631600382213$

$-3.47229716602848$

$-4.64468776127397$

$-0.90552119667062$

0.38204284130940

0.28578453272161

2. 74058852556602

3. 45800602448001

4. 95692256534562

3. 82598405848368

7. 61044815836472

8.88808961216845

8.93725864647202

11.48197459329642

7. 55803968889876

8.79187690905437

4.89157341028701

3. 75184909488129

$-1.24327766585230$

$-3.17853210356260$

$-3.89282922900139$

$-4.52150205948151$

$-6.33520992305827$

$-3.99943051067340$ h

C

C

C

C

C

$\mathrm{h}$

$\mathrm{h}$

$\mathrm{h}$

C

h

C

$\mathrm{h}$

C

$\mathrm{h}$

C

h

C

$\mathrm{h}$

C

$\mathrm{h}$

C

$\mathrm{h}$

C

$\mathrm{h}$

C

h

C

h

b

C

C

f

C

f

C

f

C

f

C

f

C

C

f

C

f

C

f

C

f

C

f

C

C

f

C

f

C 
$-7.29698619784161$

$-7.29321014326138$

$-9.74416936086571$

$-5.95154713916243$

$-7.27675161912960$

7. 90193409057407

8.38665311693545

8.94549088655023

7.21757090209383

10.36355520452301

9.67651888214689 send
8. 29864617093900

5. 05428087531227

5. 51130267509380

3. 14763274421682

1. 81528159399768

3. 62842280865790

5. 68999222054410

8. 28737736313791

9. 21641217496373

8.32904006284678

9.33950753100853
$-5.26106549825125$

$-2.11432251893467$

$-1.58922396111298$

$-0.83854413887681$

0.91081817903480

0.34807440144717

0.91390459756025

1. 62853137622837

2. 30417050741081

3. 14202291608255

$-0.00340860502148$ f

C

f

C

f

n

$\mathrm{C}$

C

$\mathrm{h}$

$\mathrm{h}$

h

\section{5. (Energy: -666.2296350 a.u.)}

$$
\begin{aligned}
& \text { \$coord } \\
& -0.00084061787388 \\
& -4.20656172423631 \\
& -3.15122338421580 \\
& -3.23412305170664 \\
& -4.34600615123460 \\
& -4.92301379279224 \\
& -4.44451983138991 \\
& -2.45312695809139 \\
& -2.61701080749859 \\
& -4.70377056959342 \\
& -5.76509969360725 \\
& 4.92016926170320 \\
& 4.12240631256152 \\
& 3.09740402981998 \\
& 3.26634719163226 \\
& 4.41576679269563 \\
& 5.76452272183083 \\
& 4.29821640182369 \\
& 2.35939022187205 \\
& 2.68617922977535 \\
& 4.83699346147715 \\
& 0.07258181315017 \\
& 0.13042637995911 \\
& 1.79514443770677 \\
& 0.16581130554326 \\
& -1.52658139913303 \\
& 0.03225376998101 \\
& -0.01798542470198 \\
& -0.06065097705146 \\
& -0.11293588712711 \\
& -1.78139385476911 \\
& 1.54182173561738 \\
& -0.13835648807631
\end{aligned}
$$
\$end
0.00107976906565

2. 29598853024981

1. 41231923425204

$-1.28654076884921$

$-2.06210685133368$

0.14874996820608

4.25760145290913

2. 58308114835745

$-2.52758891548176$

$-3.99582296062060$

0.19170404252376

$-0.33099422719003$

$-2.29324642106262$

$-1.13987877917772$

1. 53796846048295

2. 02990312424097

$-0.59450968871067$

$-4.30770234887218$

$-2.12197380534521$

2. 94820758386548

3. 88066566885236

3. 30542513637984

6.06202308963511

6.96183465358079

6.45346060601511

7. 01826133143135

1.19385868845813

$-1.32030014149405$

$-3.39463604979169$

$-6.16628057991267$

$-7.04116509739337$

$-7.09544730542919$

$-6.61600246446837$
$-0.12492529817556$

$-1.34927898712180$

$-3.65672292169533$

$-3.63264430336851$

$-1.31209708408181$

0.11266366067448

$-0.80832479887411$

$-5.18770434414065$

$-5.14301105610164$

$-0.73641223636418$

1.98092713974598

0.07336725461820

$-1.59617526158342$

$-3.79513065981922$

$-3.49258690444200$

$-1.11214039569022$

1.92218289632907

$-1.26427241491315$

$-5.43577994073895$

$-4.86302408501215$

$-0.34131813591915$

2. 87355941833040

3.45082711484094

2. 58622935259961

5.49169608428442

2.63354588501733

4.14552530433793

4.10850764712433

2. 77749122585624

3. 27384023742198

2. 39132631377061

2. 42127259842892

5. 30302453542022 zr

$\mathrm{C}$

C

C

C

C

$\mathrm{h}$

$\mathrm{h}$

$\mathrm{h}$

$\mathrm{h}$

$\mathrm{h}$

c

C

C

C

C

$\mathrm{h}$

$\mathrm{h}$

$\mathrm{h}$

$\mathrm{h}$

$\mathrm{h}$

c

C

$\mathrm{h}$

$\mathrm{h}$

$\mathrm{h}$

c

C

C

C

$\mathrm{h}$

h

h

\section{$\mathbf{C H}_{3} \mathbf{C N} \cdot \mathbf{B}\left(\mathbf{C}_{6} \mathbf{F}_{5}\right)_{3}$. (Energy: -2339.0881102 a.u.)}

\$coord

$$
\begin{aligned}
& -2.65447033856168 \\
& -2.56471734348834 \\
& -4.89046780722215
\end{aligned}
$$

$$
\begin{array}{r}
-1.67200443574764 \\
0.24428252899463 \\
1.45455061509601
\end{array}
$$

$-3.44049028059451$

$-1.59017288162001$

$-1.11044853970135$
C

C

C 
$-7.14870309085922$

$-7.13799154651499$

$-4.87273194452650$

$-0.00055164405624$

$-0.00767323128615$

$-0.01715938457749$

$-0.02937870433841$

$-5.03756171458857$

$-9.30485446344079$

$-9.26837554479857$

$-4.84624286460814$

2.66048123325477

3. 41474710639793

5.65495709069836

7. 24201574591240

6.56125597899344

4. 29895842083535

1. 91658169901755

6.27360205896831

9.38872928595039

8.05719859172205

3. 74254745770776

$-0.09339779130824$

1. 46922182983361

1.48881241845956

$-0.09930096202454$

$-1.67300982021744$

$-1.63155021537634$

3. 10502226369407

3. 01738943958895

$-0.11488884399946$

$-3.18442161740746$

$-3.14821881330798$

0.67640880711185

$-1.96255810016738$

1.18843104941155

$-0.57117126881615$ \$end
0.89155853413631

$-1.02041652711831$

$-2.30670280849230$

0.99628717136067

4.05517025592870

6. 23217242365994

8. 97288214028794

3. 27639922947386

2. 13234899855224

$-1.60426136938890$

$-4.14735877649703$

0.25058911151713

1. 47272080047855

0.90970819132086

$-1.01540780164759$

$-2.31348610229627$

$-1.67805419992052$

3. 30549405447454

2. 16133444255227

$-1.60027678973312$

$-4.16743658298702$

$-3.06448082085235$

0.24607669646042

1. 47144956143635

0.90560529042284

$-1.02411346369187$

$-2.32731335423278$

$-1.68824251599194$

3. 31054706873893

2. 16131525306669

$-1.61037107681281$

$-4.18693476830781$

$-3.07781163467712$

9. 68134984521377

9.66405868939809

9.67528193116964

$-3.04630581579324$
$-2.39373234937783$

$-4.24308432705622$

$-4.77325209463198$

0.00073312953995

0.00158871163299

0.00543829686986

0.01173571582494

0.71273498808124

$-1.85497871271382$

$-5.49327452614366$

$-6.52674281061626$

$-1.42470852552449$

$-3.66993010057992$

$-4.98471125212669$

$-4.06199426872341$

$-1.84417664770288$

$-0.58802116988268$

$-4.70025865141416$

$-7.11297327040821$

$-5.28352666740994$

$-0.95604407144733$

1. 49110632755937

3. 01698953430715

4. 79467867422743

7. 39152482882662

8.30227726274310

6.60147565964556

4. 01510196746237

4. 01526312037441

8.99330227512948

10.77182483079639

7.45071680252465

2.49085149476854

$-1.80572166856464$

0.30922300048965

1. 53717957228958

$-4.01091481127394$
C

C

C

b

$\mathrm{n}$

C

C

$\mathrm{f}$

$\mathrm{f}$

$\mathrm{f}$

f

C

C

C

C

C

C

f

$\mathrm{f}$

$\mathrm{f}$

$\mathrm{f}$

f

C

C

C

C

C

C

f

$\mathrm{f}$

$\mathrm{f}$

$\mathrm{f}$

f

$\mathrm{h}$

$\mathrm{h}$

$\mathrm{h}$

f

\section{8. (Energy: -2872.5218465 a.u.)}

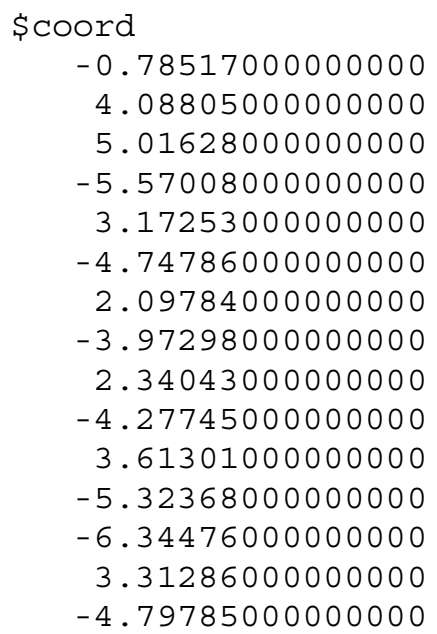

$\begin{array}{rl}0.45464700000000 & \mathrm{zr} \\ 1.05251000000000 & \mathrm{C} \\ 1.12777000000000 & \mathrm{~h} \\ 1.22236000000000 & \mathrm{C} \\ 3.16259000000000 & \mathrm{C} \\ 2.53813000000000 & \mathrm{C} \\ 2.24953000000000 & \mathrm{C} \\ 0.70579600000000 & \mathrm{C} \\ -0.44653100000000 & \mathrm{C} \\ -1.73973000000000 & \mathrm{C} \\ -1.17138000000000 & \mathrm{C} \\ -1.41111000000000 & \mathrm{C} \\ 2.07585000000000 & \mathrm{~h} \\ 5.13242000000000 & \mathrm{~h} \\ 4.56836000000000 & \mathrm{~h}\end{array}$


1. 28169000000000

$-3.28846000000000$

1. 77636000000000

$-3.88059000000000$

4.13591000000000

$-5.79604000000000$

$-0.43021000000000$

$-0.46200600000000$

$-0.31864400000000$

$-0.24668900000000$

1.34900000000000

$-1.97832000000000$

$-0.46630700000000$

$-0.65809900000000$

0.08298360000000

$-0.84636700000000$

$-1.16320000000000$

0.89181500000000

$-2.44676000000000$

0.36402600000000

2. 78355000000000

$-2.30371000000000$

2.35190000000000

2.49729000000000

0.58318900000000

$-1.45745000000000$

$-1.51451000000000$

4.26324000000000

4.45830000000000

0.70768600000000

$-3.31726000000000$

$-3.57878000000000$

$-2.30634000000000$

$-4.29036000000000$

$-6.43873000000000$

$-6.54390000000000$

$-4.49887000000000$

$-0.27523700000000$

$-4.16086000000000$

$-8.36284000000000$

$-8.59319000000000$

$-4.78609000000000$

4.87153000000000

7.20288000000000

7.53761000000000

5.51732000000000

3.20339000000000

4.69605000000000

9.08314000000000

9.74812000000000

5.79662000000000

1.34726000000000
$-12.33180000000000$

$-12.89490000000000$

$-12.36030000000000$

$-10.79430000000000$

$-8.04855000000000$

$-6.10769000000000$

$-4.76385000000000$

$-3.00629000000000$

$-4.44118000000000$

$-2.44141000000000$

$-5.38132000000000$

$-5.28867000000000$

$-0.59833100000000$

$-2.58150000000000$

2. 39007000000000

$-3.47542000000000$

$-5.53699000000000$

$-3.02186000000000$

$-2.52441000000000$

3. 75186000000000

2. 28016000000000

3. 75676000000000

5.35137000000000

6.44913000000000

5.97278000000000

4.41370000000000

3.37377000000000

5.94720000000000

7. 94419000000000

6.98868000000000

3. 92500000000000

1. 90207000000000

6.39544000000000

7. 72632000000000

6.39334000000000

3.76396000000000

2. 52367000000000

7. 78010000000000

10.24630000000000

7.62049000000000

2.45328000000000

$-0.03551550000000$

0.99857100000000

0.65297400000000

1.63950000000000

2. 91817000000000

3.19405000000000

$-0.03744120000000$

$-0.64855600000000$

1.33735000000000

3.85281000000000

4.37869000000000
3.39372000000000

1.08503000000000

$-1.70432000000000$

$-3.53555000000000$

$-3.07717000000000$

$-2.89877000000000$

$-2.75690000000000$

$-0.95810500000000$

$-5.57604000000000$

$-6.12846000000000$

$-6.38962000000000$

$-6.50058000000000$

0.36902200000000

1.88902000000000

$-0.07440990000000$

4.56471000000000

4.81385000000000

5.60416000000000

5.47703000000000

2.74829000000000

$-1.68547000000000$

$-1.56739000000000$

3. 52185000000000

5.94565000000000

7. 72666000000000

7.04328000000000

4.59708000000000

1.92499000000000

6.57547000000000

10.05480000000000

8.71954000000000

4.05575000000000

$-1.95345000000000$

$-3.11627000000000$

$-3.94227000000000$

$-3.59281000000000$

$-2.42344000000000$

$-1.22307000000000$

$-3.45019000000000$

$-5.06305000000000$

$-4.35848000000000$

$-2.16410000000000$

$-0.63502600000000$

$-1.85013000000000$

$-4.29537000000000$

$-5.45130000000000$

$-4.15124000000000$

1.73619000000000

$-0.71698100000000$

$-5.51439000000000$

$-7.80221000000000$

$-5.46358000000000$ $\mathrm{h}$

$\mathrm{h}$

$\mathrm{h}$

h

$\mathrm{h}$

$\mathrm{h}$

C

C

C

$\mathrm{h}$

$\mathrm{h}$

$\mathrm{h}$

C

C

b

C

$\mathrm{h}$

h

$\mathrm{h}$

C

C

C

C

C

C

C

C

f

$\mathrm{f}$

$\mathrm{f}$

$\mathrm{f}$

$\mathrm{f}$

C

C

C

C

C

$\mathrm{f}$

f

f

C

C

C

C

C

f

f

$\mathrm{f}$

$\mathrm{f}$

f 
9. (Energy: -3005.2999921 a.u.)

\begin{tabular}{|c|c|c|c|}
\hline ord & & & \\
\hline-0.42759318944404 & -9.49067224012638 & -2.65048520584761 & $\mathrm{n}$ \\
\hline-0.32849843173886 & -10.39710751108039 & -4.64512238656120 & $\mathrm{C}$ \\
\hline-0.19782009496616 & -11.50546527508607 & -7.15949317069566 & C \\
\hline 1.42675003253525 & -12.78874607985400 & -7.29315953115002 & $\mathrm{~h}$ \\
\hline-1.92793554129775 & -12.57698373465469 & -7.56088710609294 & $\mathrm{~h}$ \\
\hline 0.00894432140386 & -10.01255545616161 & -8.58495078444895 & $\mathrm{~h}$ \\
\hline-0.54199963943896 & -7.24764738346369 & 1.13454035671555 & $\mathrm{zr}$ \\
\hline 4.03844100189190 & -6.46746309647513 & 2.85629371693912 & $\mathrm{C}$ \\
\hline 4.71463278541083 & -4.53226697574368 & 2.83379838068434 & $\mathrm{~h}$ \\
\hline-4.92211945128371 & -5.90964597454401 & 2.95408604439874 & $\mathrm{C}$ \\
\hline 2.71296295997990 & -7.67364909284697 & 4.88391329949853 & C \\
\hline-4.03766887414202 & -7.85683222052630 & 4.60860862417042 & C \\
\hline 2.23363109361371 & -10.22174943426481 & 4.16706036245889 & $\mathrm{C}$ \\
\hline-4.04293521123235 & -10.17195960006632 & 3.24103373847510 & $\mathrm{C}$ \\
\hline 3.15180618194458 & -10.56125835384867 & 1.67271746860393 & $\mathrm{C}$ \\
\hline-4.89891915079617 & -9.64844790723004 & 0.74384324312300 & $\mathrm{C}$ \\
\hline 4.28864142218665 & -8.23312569061118 & 0.86516787572798 & $\mathrm{C}$ \\
\hline-5.44610408308698 & -7.01387742438704 & 0.56297955756532 & C \\
\hline-5.18639510722445 & -3.93484784025237 & 3.43287338722009 & $\mathrm{~h}$ \\
\hline 2.29616217114406 & -6.84489519230504 & 6.71045074583952 & $\mathrm{~h}$ \\
\hline-3.55676766156020 & -7.63312050323078 & 6.58975301032733 & $\mathrm{~h}$ \\
\hline 1.30047536593895 & -11.64012538184449 & 5.31372522995693 & $\mathrm{~h}$ \\
\hline-3.52854992031799 & -12.01520880811742 & 3.97373076972918 & $\mathrm{~h}$ \\
\hline 3.08875776664558 & -12.30574607471136 & 0.59622125992410 & $\mathrm{~h}$ \\
\hline-5.15603204185935 & -11.03301063546106 & -0.74561243206989 & $\mathrm{~h}$ \\
\hline 5.20298996430611 & -7.88931136932496 & -0.93613113607296 & $\mathrm{~h}$ \\
\hline-6.15715128716135 & -6.01996086708011 & -1.08076738226519 & $\mathrm{~h}$ \\
\hline-0.27231752774012 & -4.30782580000302 & -1.61705327102128 & C \\
\hline-0.25478972655356 & -2.61383284672974 & 0.30633783045516 & C \\
\hline-0.31698173040689 & -3.61264666835816 & -4.38050120539147 & C \\
\hline-0.16940164727662 & -1.56876059688291 & -4.69724847901267 & $\mathrm{~h}$ \\
\hline 1.25418388664146 & -4.51293553840781 & -5.40919195677290 & $\mathrm{~h}$ \\
\hline-2.07930436580918 & -4.25508417000151 & -5.28722733340901 & $\mathrm{~h}$ \\
\hline-0.29566547036113 & 0.07237200682496 & 1.00257249145314 & C \\
\hline-0.29798208781123 & -1.56526843318590 & 2.89203936742204 & C \\
\hline 0.08603830281536 & 2.90332320481128 & -0.11430842279705 & $\mathrm{~b}$ \\
\hline-0.23497245743559 & -1.80162075181675 & 5.70329437854044 & C \\
\hline-1.09144770896611 & -3.55056951035943 & 6.39790420300686 & $\mathrm{~h}$ \\
\hline 1.72442868623052 & -1.71840032873209 & 6.39111498475004 & $\mathrm{~h}$ \\
\hline-1.26179916424953 & -0.19240373737025 & 6.50729127751056 & $\mathrm{~h}$ \\
\hline 0.24988349184079 & 4.88298241412867 & 2.32466632031255 & $\mathrm{C}$ \\
\hline 2.80235079301531 & 2.58357033188060 & -1.67754639568305 & $\mathrm{C}$ \\
\hline-2.35009995751696 & 3.82035882569689 & -1.85348598759275 & $\mathrm{C}$ \\
\hline 2.08541793574922 & 6.78093175615287 & 2.68888605522098 & C \\
\hline 2.12973778537332 & 8.38903061564490 & 4.81396197091984 & C \\
\hline 0.26136682715529 & 8.14903040721116 & 6.68915531944490 & C \\
\hline-1.63193703313024 & 6.30902771730061 & 6.39595425568992 & C \\
\hline-1.61442698663136 & 4.78147043723862 & 4.22254821527417 & C \\
\hline 3.95340727568001 & 7.15279187881372 & 0.97638137363539 & $\mathrm{f}$ \\
\hline 3.94659331727319 & 10.15649317780563 & 5.06886551526045 & $\mathrm{f}$ \\
\hline 0.29200259826096 & 9.65016484497260 & 8.74264459760089 & $\mathrm{f}$ \\
\hline-3.43022205279388 & 6.01601854224172 & 8.18218817471659 & $\mathrm{f}$ \\
\hline-3.54899460385319 & 3.07132897224558 & 4.04524233574810 & $\mathrm{f}$ \\
\hline-2.36508173614145 & 6.26929132392060 & -2.91088916683557 & C \\
\hline-4.38975462005340 & 7.27182190083211 & -4.31038588332788 & C \\
\hline
\end{tabular}


$-6.58657632364398$

$-6.67588548341174$

$-4.59114494603909$

$-0.30183566611936$

$-4.26241649734705$

$-8.55896880492489$

$-8.76168880964235$

$-4.86338632953343$

5.01449459457882

7. 37619620175717

7. 60223960229661

5.45120807235503

3. 11987317707742

4.93543075882089

9. 40168006832297

9. 84131748780007

5.62269551882460

1.12996633700691
5.80790441110588

3. 35817641056806

2. 44238392731599

7. 77148847164918

9. 61505366075679

6.72909998584052

1. 92048464712055

0.04885792589183

1. 88911562843861

1. 47078782833491

1. 72843183074821

2. 36567081205377

2. 74698920474033

1. 54308130912014

0.79609095131841

1. 34066814176732

2. 56560569756595

3. 23482439203288
$-4.64681792531225$

$-3.62603453592160$

$-2.24245126403544$

$-2.65275445339975$

$-5.30100800736690$

$-5.96505228870395$

$-3.94323384765024$

$-1.30083220243807$

$-0.35890145191795$

$-1.49725509424125$

$-4.13356694225415$

$-5.55384145042212$

$-4.31606812803044$

2. 20275417547219

$-0.10329484928532$

$-5.27879717360056$

$-8.08860819987447$

$-5.87086721512059$
C

C

C

f

$\mathrm{f}$

f

$\mathrm{f}$

f

C

C

C

C

C

f

f

$\mathrm{f}$

f

f

\$end 


\section{Kinetic study by NMR (600 MHz Varian UnityPlus spectrometer)}

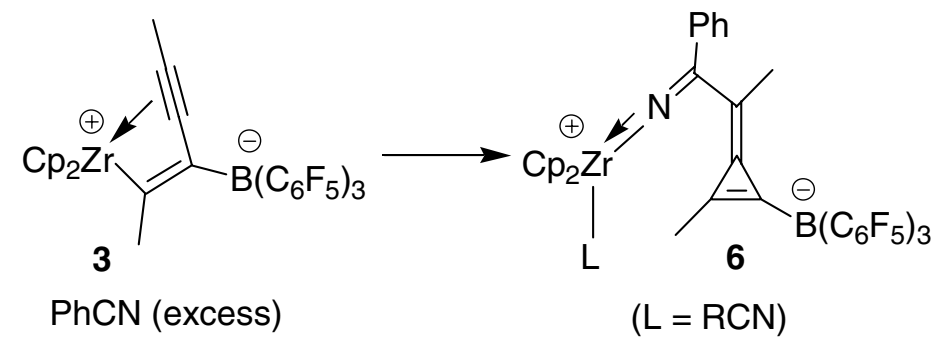

Basic equations:

Formation of the product: $\quad I=A_{1} \exp (-t / t a u)+A_{3}$

$$
\begin{aligned}
& {[\mathrm{P}]_{\mathrm{t}}=[\mathrm{P}]_{0} \exp \left(-\mathrm{k}_{\exp } \mathrm{t}\right)} \\
& -\mathrm{t} / \mathrm{tau}=-\mathrm{k}_{\mathrm{obs}} \mathrm{t} \\
& \mathrm{k}_{\text {obs }}=1 / \mathrm{tau} \\
& \mathrm{k}_{\text {chem }}=\mathrm{k}_{\mathrm{obs}} /[\mathrm{PhCN}] \\
& \Delta \mathrm{G}_{\text {chem }}^{\ddagger}=-\mathrm{R} \mathrm{T} \ln \left(\left(\mathrm{k}_{\text {chem }} \mathrm{h}\right) /\left(\mathrm{k}_{\mathrm{b}} \mathrm{T}\right)\right) \\
& \mathrm{R}=8.31451\left(\mathrm{~m}^{2} \cdot \mathrm{kg}\right) /\left(\mathrm{s}^{2} \cdot \mathrm{K} \cdot \mathrm{mol}\right) \\
& \mathrm{k}_{\mathrm{b}}=1.38065810^{-23} \mathrm{~J} / \mathrm{K} \\
& \mathrm{h}=6.626075510^{-34} \mathrm{~J} \mathrm{~s}
\end{aligned}
$$

Consumption of the educt: $\quad I=-A_{1} \exp (-t / t a u)+A_{3}-A_{1}$

\section{Kinetic experiments:}

Observation of the decreasing of $\delta^{1} \mathrm{H}$ (educt) $=5.67,5.38$ (each 5H, each $\mathrm{Cp}$ )

Observation of the increasing of $\delta^{1} \mathrm{H}$ (product) $=5.49(10 \mathrm{H}, \mathrm{Cp})$

3 and a defined amount of ferrocene (as an inert, internal standard) were dissolved in $0.6 \mathrm{ml}$ of toluene- $\mathrm{d}_{8}$ at room temperature. Subsequently the NMR tube was cooled at $-78^{\circ} \mathrm{C}$ and $7.5 \mu 1$ of benzonitrile was added slowly. Directly, after careful mixing of the solution in the cooling bath, the NMR tube was injected into the spectrometer that was cooled at $-50^{\circ} \mathrm{C}$. The experiment was started at $-30^{\circ} \mathrm{C}$.

Series A:

243K; [3] (4.8 mg in $0.6 \mathrm{ml}$ toluene- $\left.\mathrm{d}_{8}\right) 0.010 \mathrm{~mol} / \mathrm{l} ;[\mathrm{PhCN}]:(7.5 \mu \mathrm{l}) 0.122 \mathrm{~mol} / 1$

Series $B$ :

243K; [3] (2.7 mg in $0.6 \mathrm{ml}$ toluene- $\left.\mathrm{d}_{8}\right) 0.006 \mathrm{~mol} / \mathrm{l} ;[\mathrm{PhCN}]:(7.5 \mu \mathrm{l}) 0.122 \mathrm{~mol} / \mathrm{l}$ 
Series $C$ :

243K; [3] (2.1 mg in $0.6 \mathrm{ml}$ toluene-d $\left.{ }_{8}\right) 0.004 \mathrm{~mol} / \mathrm{l}$; [PhCN]: $(7.5 \mu \mathrm{l}) 0.122 \mathrm{~mol} / \mathrm{l}$

Series D:

243K; [3] (2.9 mg in $0.6 \mathrm{ml}$ toluene-d8) $0.006 \mathrm{~mol} / \mathrm{l}$; [PhCN]: (7.5 $\mu \mathrm{l}) 0.122 \mathrm{~mol} / \mathrm{l}$

\begin{tabular}{|c|c|c|c|c|}
\hline & series A & series B & series $\mathrm{C}$ & seriesD \\
\hline $\operatorname{tau}(5.67)$ & 2542 & 2296 & 2253 & 2383 \\
\hline $\mathrm{k}_{\mathrm{obs}}(5.67)$ & $3.933910^{-4}$ & $4.355410^{-4}$ & $4.438510^{-4}$ & $4.196410^{-4}$ \\
\hline $\mathrm{k}_{\text {chem }}(5.67)$ & $3.232410^{-3}$ & $3.578810^{-3}$ & $3.647110^{-3}$ & $3.448110^{-3}$ \\
\hline$\Delta \mathrm{G}_{\text {chem }}^{\ddagger}(5.67)$ & 16.9 & 16.8 & 16.8 & 16.9 \\
\hline $\operatorname{tau}(5.38)$ & 2698 & 2357 & 2108 & 2361 \\
\hline $\mathrm{k}_{\mathrm{obs}}(5.38)$ & $3.706410^{-4}$ & $4.242610^{-4}$ & $4.743810^{-4}$ & $4.235510^{-4}$ \\
\hline $\mathrm{k}_{\text {chem }}(5.38)$ & $3.555610^{-3}$ & $3.486210^{-3}$ & $3.898010^{-3}$ & $3.480310^{-3}$ \\
\hline$\Delta \mathrm{G}_{\text {chem }}^{\ddagger}(5.38)$ & 16.9 & 16.8 & 16.8 & 16.8 \\
\hline $\operatorname{tau}(5.49)$ & 2311 & 2069 & not used & 2076 \\
\hline $\mathrm{k}_{\mathrm{obs}}(5.49)$ & $4.327110^{-4}$ & $4.833310^{-4}$ & & $4.817010^{-4}$ \\
\hline $\mathrm{k}_{\mathrm{chem}}(5.49)$ & $3.555610^{-3}$ & $3.971410^{-3}$ & & $3.958110^{-3}$ \\
\hline$\Delta \mathrm{G}_{\text {chem }}^{\ddagger}(5.49)$ & 16.8 & 16.8 & & 16.8 \\
\hline
\end{tabular}


$\frac{0}{n}$
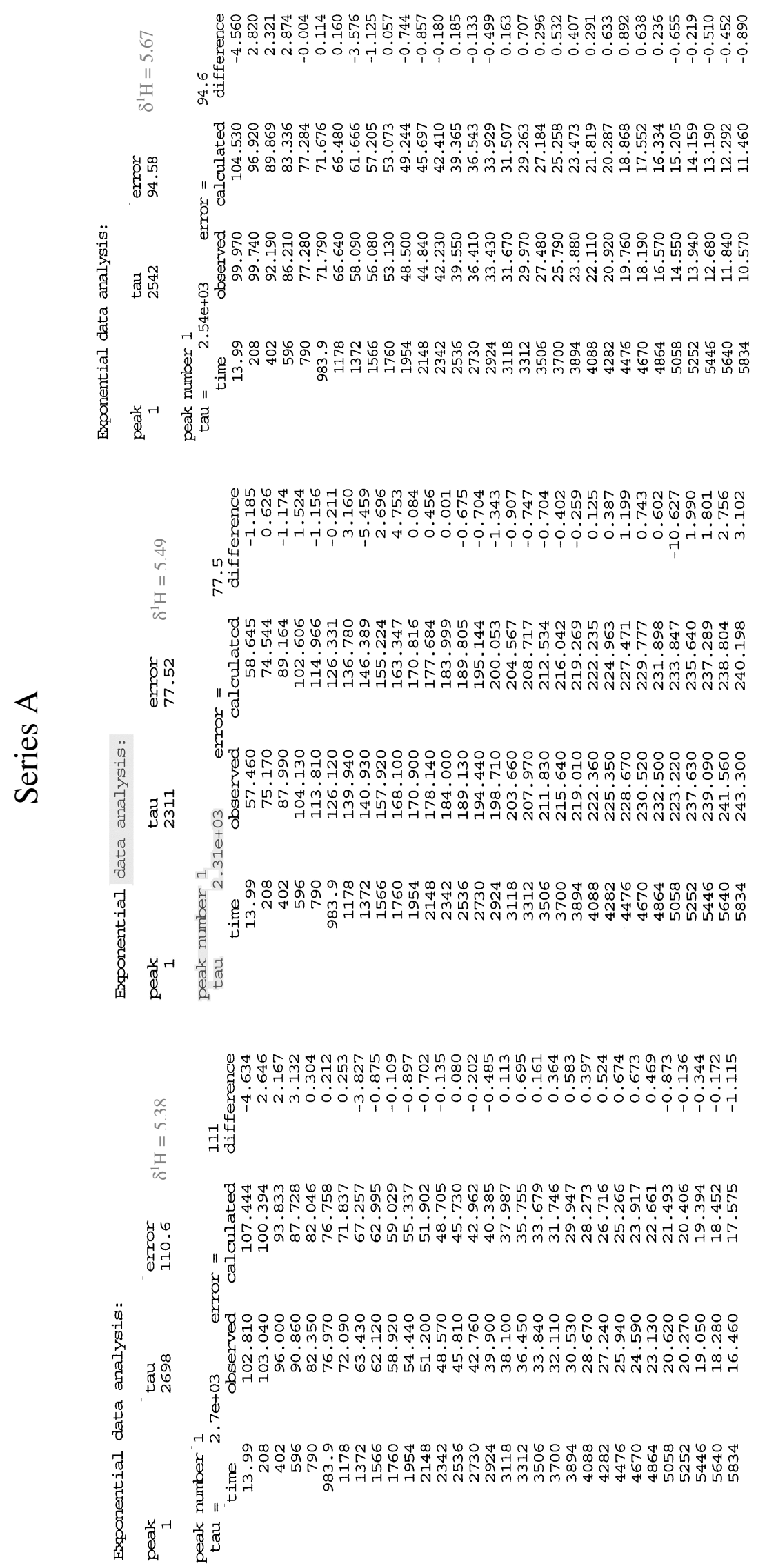
$\frac{N}{n}$
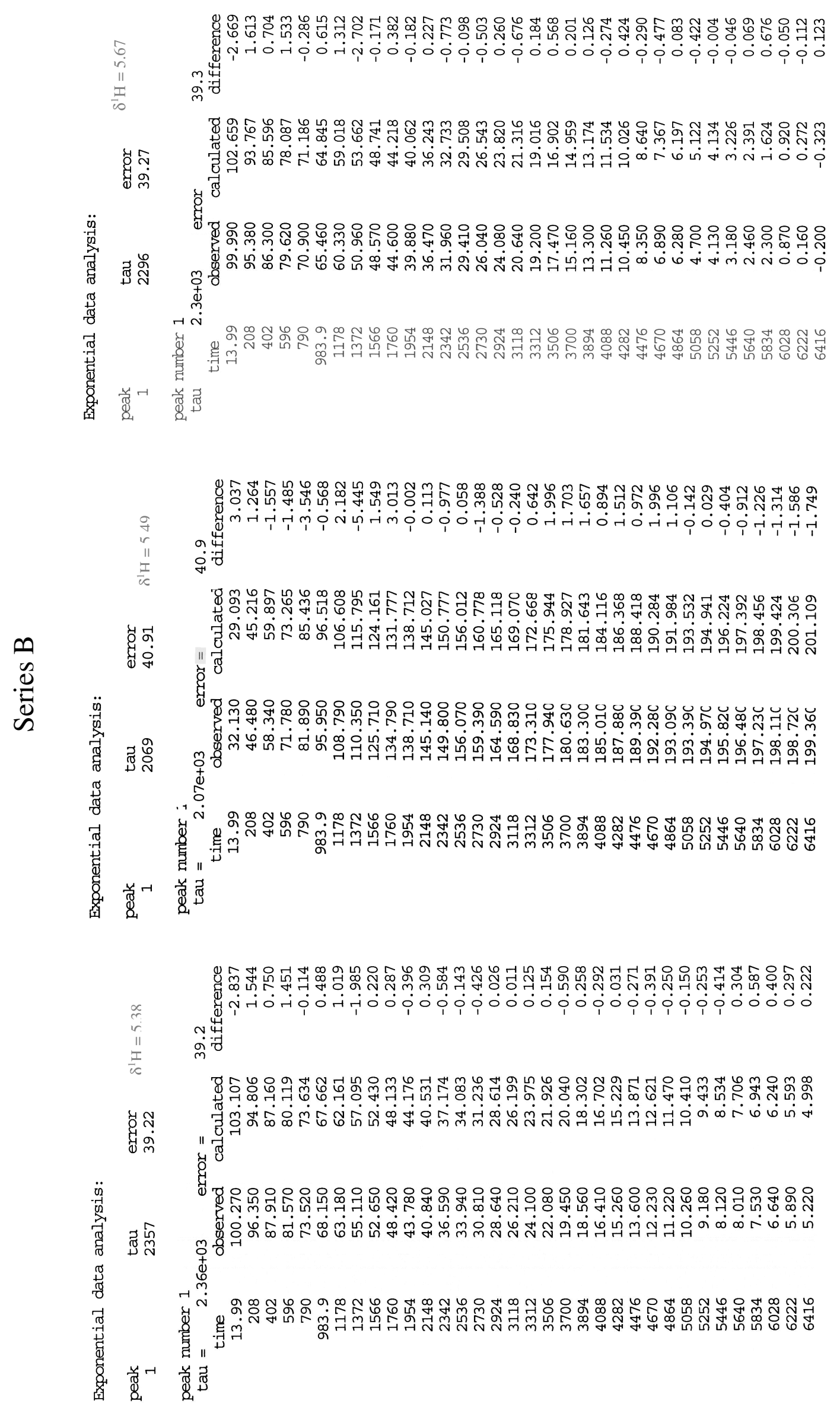

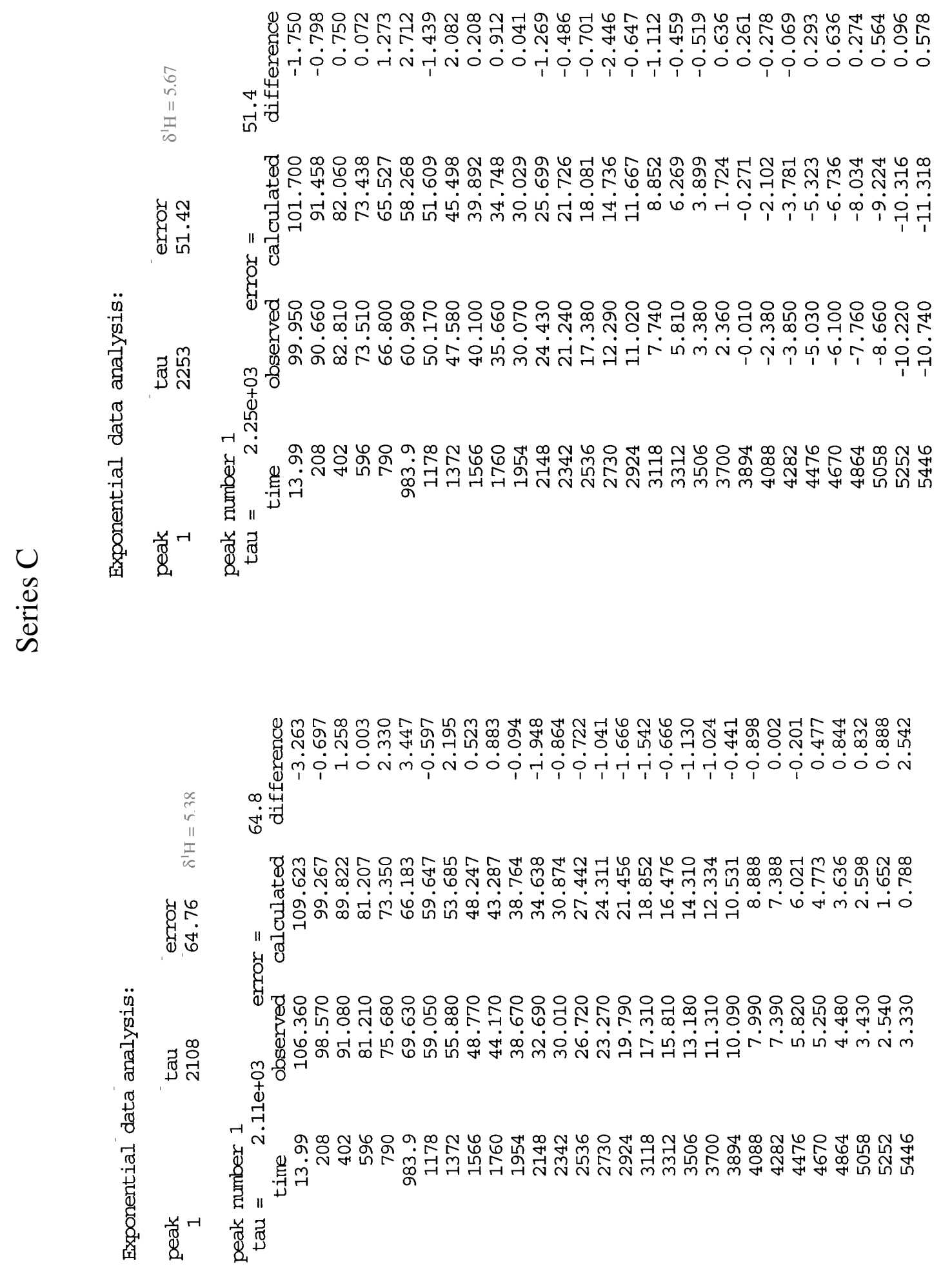

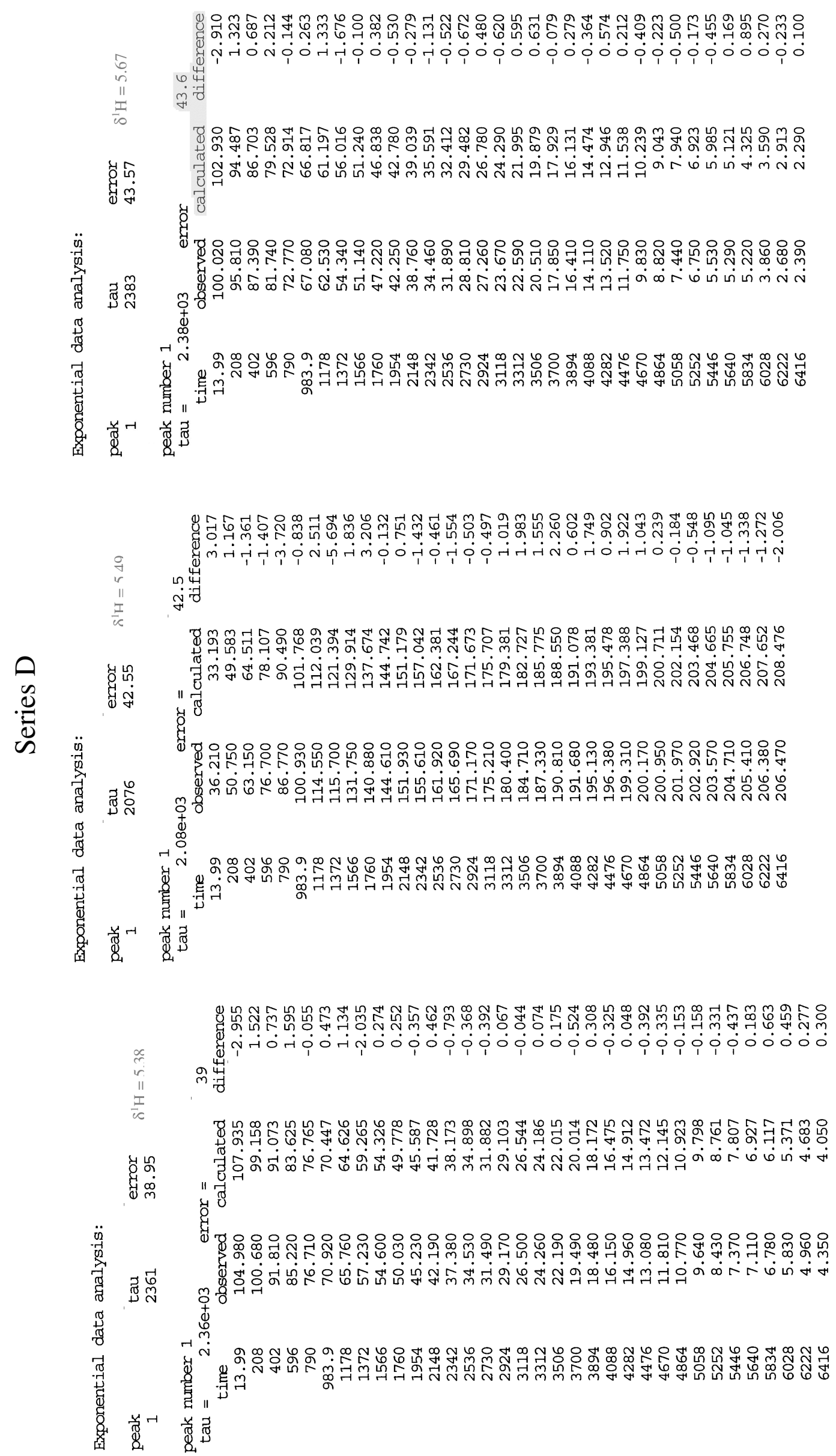